\title{
ON PHI-FAMILIES
}

CHARLES E. WATTS

1. Introduction. The purpose of this note is to show that the notion of sections with support in a phi-family in the Cartan version of the Leray theory of sheaves can be avoided by the following expedient. One uses the phi-family to construct a new space in the manner of a one-point compactification. Then a sheaf on the original space is shown to yield a new sheaf on the new space whose cohomology (with unrestricted supports) is that of the original sheaf with restricted supports. A partial generalization to the Grothendieck cohomology theory is given.

2. Phi-families. A family $\mathcal{F}$ of subsets of a topological space $X$ is a family of supports [1] provided:

(I) each member of $\mathcal{F}$ is closed;

(II) if $F \in \mathcal{F}$, then each closed subset of $F$ is $\in \mathcal{F}$;

(III) if $F_{1}, F_{2} \in \mathcal{F}$, then $F_{1} \cup F_{2} \in \mathcal{F}$.

Given a family of supports $\mathcal{F}$, we choose an object $\infty \notin X$, and define $X^{\prime}=\bigcup F, X^{*}=X^{\prime} \cup\{\infty\}$. We then topologize $X^{*}$ by saying that a subset $U$ of $X^{*}$ is open iff either $U$ is open in $X^{\prime}$ or else $X^{*}-U \in \mathcal{F}$. It is readily verified that these open sets in fact form a topology for $X^{*}$ and that the inclusion $X^{\prime} \subset X^{*}$ is a topological imbedding.

The family of supports $\mathcal{F}$ is a phi-family [3] provided also:

(IV) each member of $\mathcal{F}$ has a closed neighborhood in $\mathcal{F}$;

(V) each member of $\mathfrak{F}$ is paracompact.

Proposition 1. If $\mathcal{F}$ is a phi-family, then $X^{*}$ is paracompact.

Proof. Let $\mathcal{u}$ be any open cover of $X^{*}$ and choose $U_{\infty} \in \mathcal{U}$ with $\infty \in U$. Then $X^{*}-U_{\infty} \in \mathcal{F}$, so we can find an open set $V$ with $X^{*}-U_{\infty}$ $\subset V \subset X^{\prime}, \bar{V} \in \mathcal{F}$. Now the sets of the form $U \cap \bar{V}, U \in \mathcal{U}$, cover $\bar{V}$; since $\bar{V}$ is paracompact there is a locally finite refinement $\mathcal{u}^{\prime}$ of this cover of $\bar{V}$. Now let $\mathcal{u}^{\prime \prime}$ be the family of all sets $U^{\prime} \cap V, U^{\prime} \in \mathcal{u}^{\prime}$. Then $\gamma=u^{\prime \prime} \cup\left\{U_{\infty}\right\}$ is a locally finite open cover of $X^{*}$, refining $\mathcal{u}$.

To show that $X^{*}$ is a Hausdorff space, let $x$ and $y$ be distinct points of $X^{*}$. If $y=\infty$, then $\{x\} \in \mathcal{F}$; we choose a neighborhood $U$ of $x$ with $\bar{U} \in \mathcal{F}$ and then $U, X^{*}-\bar{U}$ are disjoint neighborhoods of $x, y$. If $x \neq \infty$ and $y \neq \infty$, we choose $U$ as before. If $y \notin \bar{U}$, then $U, X^{*}-\bar{U}$ are disjoint neighborhoods of $x, y$. If $y \in \bar{U}$, then since $\bar{U}$ is Hausdorff, we can choose open subsets $V, W$ of $X^{\prime}$ with $x \in V, y \in W, V \cap W \cap \bar{U}$ $=\varnothing$. Then $V \cap U, W \cap\left(X^{*}-\bar{U}\right)$ are disjoint neighborhoods of $x, y$.

Received by the editors October 24, 1958. 
As examples of families of supports, we cite the following:

(1) The compact subsets of a locally compact space;

(2) The bounded closed subsets of a metric space;

(3) The closed subsets of a space which do not meet a fixed subset $A$. This will be a phi-family if $A$ is closed and the whole space $X$ is paracompact. $X^{*}$ is the quotient space of $X$ obtained by identifying $A$ to a point. Such a family may be used to define the relative cohomology $H(X, A ; \mathfrak{Q})$ with coefficients in a sheaf $\mathfrak{Q}$, in a manner described in the next section.

3. Applications to sheaf theory. Let $\mathcal{F}$ be a family of supports for a space $X$ and let $Q$ be a sheaf of rings or modules over $X$. We form the restriction $a \mid X^{\prime}$ of $a$ to $X^{\prime}$ and then construct a sheaf $a^{*}$ over $X^{*}$ by defining $Q^{*}\left|X^{\prime}=a\right| X^{\prime}$ and letting the stalk $A_{\infty}^{*}$ of $Q^{*}$ over $\infty$ be zero. A neighborhood of $A_{\infty}^{*}$ consists of the zeroes of stalks over a neighborhood of $\infty$. That the sheaf $a^{*}$ is well determined by these data is easily verified. A map $f: a \rightarrow B$ of sheaves over $X$ determines a map $f^{*}: a^{*} \rightarrow a^{*}$ of sheaves over $X^{*}$ in an obvious way, and the following proposition is immediate.

Proposition 2. The correspondence $a \rightarrow Q^{*}, f \rightarrow f^{*}$ is an exact functor from the category of sheaves over $X$ to that of sheaves over $X^{*}$.

Proposition 3. If $\mathcal{F}$ is a phi-family and if $a$ is $\mathcal{F}$-fine, then $a^{*}$ is fine.

Proof. Let $\mathcal{U}^{*}$ be a locally finite open cover of $X^{*}$. We may assume that $\mathfrak{u}^{*}=\mathfrak{u}^{\prime} \cup\left\{X^{*}-F\right\}$, where $\mathfrak{u}^{\prime}$ is a cover of $X^{\prime}$ and $F \in \mathcal{F}$, since such covers are clearly cofinal among all covers of $X^{*}$. Then $u$ $=\mathcal{U}^{\prime} \cup\{X-F\}$ is a locally finite $\mathcal{F}$-cover of $X$. If $\left\{l_{i}\right\}$ are endomorphisms of $a$ making up a partition of unity for $Q$ and $u$, then the endomorphisms $\left\{l_{i}^{*}\right\}$ make up a partition of unity for $a^{*}$ and $u^{*}$. Hence $a^{*}$ is fine.

Proposition 4. If $\mathcal{F}$ is any family of supports on a space $X$, and if $a$ is any sheaf over $X$, then there is a natural isomorphism

$$
\Gamma\left(X^{*} ; Q^{*}\right) \approx \Gamma \mathscr{F}(X ; \mathfrak{Q}),
$$

where $\Gamma_{\mathscr{F}}(X ; \mathfrak{Q})$ is the module of sections of a over $X$ with supports in $\mathcal{F}$.

The proof of Proposition 4 is immediate.

Theorem A. Let $\mathcal{F}$ be a phi-family on a space $X$, a a sheaf over $X$. Then there are natural isomorphisms, for each $p \geqq 0$,

$$
H^{p}\left(X^{*} ; Q^{*}\right) \approx H_{\mathfrak{F}}^{p}(X ; Q) .
$$


Proof. The preceding propositions shows that the modules $H^{p}\left(X^{*} ; Q^{*}\right)$ and their associated maps satisfy the Cartan axioms [3] for the $\mathcal{F}$-cohomology of $Q$.

We do not know whether or not the analog of Theorem A holds for arbitrary families of supports using the Grothendieck cohomology theory of sheaves $[\mathbf{1} ; \mathbf{2}]$. However, the following result is true.

THEOREM B. If $\mathcal{F}$ is any family of supports on a space $X$ such that $U_{\mathcal{F}}=X$, and if $Q$ is any sheaf on $X$, then there are natural isomorphisms

$$
H^{p}\left(X^{*} ; Q^{*}\right) \approx H \stackrel{F}{p}(X ; \propto),
$$

where the cohomology modules are defined in the sense of Grothendieck.

The proof reduces to the easy verification that, under the assumption on $\mathcal{F}$, if $Q$ is injective, then so is $Q^{*}$.

\section{REFERENCES}

1. R. Godement, Theorie des faisceaux, Paris, 1958.

2. A. Grothendieck, Sur quelques points d'algebre homologique, Tôhoku Math. J. (2) vol. 9 (1957) pp. 119-221.

3. Seminaire Henri Cartan, 1950-1951, 2d ed., Paris, 1955.

The University of Chicago 